\title{
Solvable non-Markovian dynamic network
}

\author{
Nicos Georgiou, ${ }^{*}$ Istvan Z. Kiss, ${ }^{\dagger}$ and Enrico Scalas ${ }^{\ddagger}$ \\ School of Mathematics and Physical Sciences, University of Sussex, Brighton BN1 9QH, United Kingdom
}

(Received 17 March 2015; revised manuscript received 2 July 2015; published 2 October 2015)

\begin{abstract}
Non-Markovian processes are widespread in natural and human-made systems, yet explicit modeling and analysis of such systems is underdeveloped. We consider a non-Markovian dynamic network with random link activation and deletion (RLAD) and heavy-tailed Mittag-Leffler distribution for the interevent times. We derive an analytically and computationally tractable system of Kolmogorov-like forward equations utilizing the Caputo derivative for the probability of having a given number of active links in the network and solve them. Simulations for the RLAD are also studied for power-law interevent times and we show excellent agreement with the Mittag-Leffler model. This agreement holds even when the RLAD network dynamics is coupled with the susceptible-infected-susceptible spreading dynamics. Thus, the analytically solvable Mittag-Leffler model provides an excellent approximation to the case when the network dynamics is characterized by power-lawdistributed interevent times. We further discuss possible generalizations of our result.
\end{abstract}

DOI: 10.1103/PhysRevE.92.042801

PACS number(s): 89.75.Hc, 02.50.Ey, 89.75.Da

\section{INTRODUCTION}

Non-Poisson temporal statistics where time intervals between isolated consecutive actions are typically not exponentially distributed seem to be the norm rather than the exception for many systems, for example, period of infectiousness [1], interorder and intertrade durations in financial markets [2], socionetworks, including emails [3,4], phone calls [5], or individual-to-individual contacts being fluid [6,7]. The absence of the robust tools and mathematical machinery of Markovian theory is the source of many challenges in modeling and analysis of non-Markovian systems. The burst in research activity that successfully combines networks and non-Markovian processes stems from the need to develop more realistic models and new analytical tools. Notable examples include studying non-Poisson dynamics of networks [8] and non-Markovian epidemics on networks [9-11].

The non-Markovian property is particularly pervasive when considering the dynamics of time-evolving networks, be it with a fast or slow time scale $[12,13]$. Deriving simple, solvable paradigmatic models can facilitate progress in developing new mathematical tools and methods for analysis and increases our understanding of the true implications of non-Markovianity for complex systems. Empirically, it turns out that many interevent distributions have power-law tails (see [14] and references therein). Therefore, it is also necessary to develop methods able to deal with such distributions.

It is now widely accepted that human contact patterns are highly dynamic and may evolve concurrently with an epidemic; many Markovian models for this setup exist [15-17]. Here we take the next step and consider a dynamic network with nonexponential waiting times with consecutive updates that are either link activation or deletion [17]. As a first step in the rigorous analysis of networks with nonMarkovian dynamics we consider a random link activationdeletion (RLAD) model that naturally leads to a stochastically

\footnotetext{
*N.Georgiou@sussex.ac.uk

†I.Z.Kiss@sussex.ac.uk

${ }^{\ddagger}$ E.Scalas@sussex.ac.uk
}

evolving network [17,18]. This model amounts to considering undirected and unweighted networks, where an event consists of selecting a link at random, independently of whether or not it is present, followed by its activation if the link is absent or deletion if the link is active. Such operations are separated by interevent times sampled from the Mittag-Leffler distribution, which allows for analytical tractability. This exactly solvable model of non-Markovian network dynamics is an important special case of a more general theory for non-Markovian processes outlined in [19] and it is related to recent outstanding developments in probability theory [20,21]. Indeed, we provide a bottom-up derivation for the master equation of some fractional birth and death processes in a finite capacity system, introduced in [20]. This allows us to compute theoretically the exact distribution of the total number of links in the network at any time and its large-time limit. We demonstrate the power of the analytical model by comparing it with simulations using more widely used power-law-distributed times. The rigorous analysis of this model, including explicit expressions for the distribution of the number of links in the network for $t \geqslant 0$, is followed by considering a Markovian susceptible-infected-susceptible (SIS) epidemic on our non-Markovian dynamic network. Finally, we briefly discuss the generalization of our method to general Markov chains with random state changes occurring according to a generic renewal process.

\section{EXACTLY SOLVABLE MODEL}

\section{A. Basic ingredients}

Consider an arbitrary graph on $N$ nodes as an initial state of the dynamics. We are interested in the number of (unique undirected) links in the network at a given time $t$. We denote this number by $X(t)$ and it takes values in $\mathcal{S}=\{0,1, \ldots, M\}$ where $M=N(N-1) / 2$, the maximal possible number of links. The time periods where $X(t)$ remains constant are called sojourn times or interevent times. We assume that sojourn times $\left\{T_{i}\right\}_{i \geqslant 1}$ are drawn independently from the family of Mittag-Leffler distributions with the parameter (or order) $\beta \in(0,1)$ [22]. Their cumulative distribution function (CDF) 
is indexed by this $\beta$ and it is given by

$$
F_{T}^{(\beta)}(t)=\mathbb{P}\{T \leqslant t\}=1-E_{\beta}\left(-t^{\beta}\right) .
$$

Here $E_{\beta}(z)$ is the Mittag-Leffler function, defined by

$$
E_{\beta}(z)=\sum_{n=0}^{\infty} \frac{z^{n}}{\Gamma(1+\beta n)},
$$

where $E_{\beta}$ is entire for all $\beta>0$. At $\beta=0$ the series converges uniformly only on a disk of radius 1 , though the function can be extended analytically on $\mathbb{C} \backslash\{1\}$. Equations (1) and (2) define a proper CDF only when $\beta \in(0,1]$. This is equivalent to the claim that, for $\beta \in(0,1], E_{\beta}\left(-t^{\beta}\right)$ is completely monotone. A $C^{\infty}[0, \infty)$ function $f(t)$ is completely monotone if $(-1)^{n} d^{n} f(t) / d t^{n} \geqslant 0$ for all non-negative integers $n$ and all $t>0$. Mainardi and Gorenflo [23] proved that, for $\beta \in$ $(0,1), E_{\beta}\left(-t^{\beta}\right)$ can be written as a mixture of exponential distributions given that

$$
E_{\beta}\left(-t^{\beta}\right)=\int_{0}^{\infty} \exp (-r t) K_{\beta}(r) d r,
$$

where

$$
K_{\beta}(r)=\frac{1}{\pi} \frac{r^{\beta-1} \sin (\beta \pi)}{r^{2 \beta}+2 r^{\beta} \cos (\beta \pi)+1}
$$

and

$$
\int_{0}^{\infty} K_{\beta}(r) d r=1 .
$$

Therefore, complete monotonicity of $E_{\beta}\left(-t^{\beta}\right)$ is an immediate corollary of Bernstein's theorem [24,25]. A direct proof that $E_{\beta}(-x)$ is completely monotone can be found in Ref. [26]. When $0<\beta<1$ these distributions are heavy tailed with infinite mean while at $\beta=1, T$ is mean 1 , exponentially distributed. This family of distributions interpolates between a stretched exponential for small $t$ and a power-law for large $t$ [23]. Namely, one has

$$
\begin{aligned}
& E_{\beta}\left(-t^{\beta}\right) \simeq \exp \left[-t^{\beta} / \Gamma(1+\beta)\right], \quad t \ll 1 \\
& E_{\beta}\left(-t^{\beta}\right) \sim \frac{\sin (\beta \pi)}{\pi} \frac{\Gamma(\beta)}{t^{\beta}}, \quad t \rightarrow \infty .
\end{aligned}
$$

Therefore, the use of these distributions is more general than it might seem at a first glance. A word of notational caution is should be noted: Here $\beta$ is the order of the polynomial decay of the survival function, but most commonly powerlaw distributions are identified by the order of decay of their densities, which in our case is $1+\beta \in(1,2)$.

Mittag-Leffler sojourn times lead to a simpler analytical treatment of non-Markovianity in the presence of extreme power-law tails than its cognate Pareto distribution. However, we do explain below how the theoretical framework developed here can be used to approximate the behavior of non-exactlysolvable systems with Pareto power-law distribution, as it is most commonly used. For this we must introduce a scaling parameter (time change) $\gamma>0$ for the waiting times: We say that a random variable $T$ is $\mathcal{D}_{\mathrm{ML}_{\gamma}}(\beta)$ distributed if and only if

$$
F_{T}^{(\beta, \gamma)}(t)=\mathbb{P}\{T \leqslant t\}=1-E_{\beta}\left(-(t / \gamma)^{\beta}\right) .
$$

For $\gamma=1$ the CDF is reduced to that of Eq. (1) and we see that $T$ is $\mathcal{D}_{\mathrm{ML}_{1}}(\beta)$ if and only if $\gamma T$ is $\mathcal{D}_{\mathrm{ML}_{\gamma}}(\beta)$.
For the rigorous derivation of the evolution equations, for clarity we restrict ourselves to the $\gamma=1$ case and remark how the equations behave with the extra scaling later. Fix a parameter $\beta \in(0,1)$. The network evolves in a semi-Markov way: Let $T_{1}, T_{2}, \ldots$ be independent $\mathcal{D}_{\mathrm{ML}}(\beta)$ times and define the partial sum

$$
S_{n}=\sum_{k=1}^{n} T_{i}, \quad n \geqslant 1 .
$$

The sequence $S_{1}, S_{2}, \ldots$ denotes the event times at which the state of the network $X(t)$ attempts to change. A change in the state means an undirected link is either deleted or activated. For extra flexibility, the model is introduced with an extra delay parameter $\alpha \in[0,1)$, so that if $\alpha \neq 0$ the number of active links may remain unchanged, even if there is an attempt of a change.

It is useful to define the embedded Markov chain for the number of links in the network $X_{n}, n \geqslant 1$, with state space $\mathcal{S}$. Initially $X_{0}=i$, as we start with $i$ present links and the number of links in the network increases, remains the same, or decreases according to the transition probabilities

$$
\begin{aligned}
& q_{k, k-1}= P_{0}\left\{X_{j+1}=k-1 \mid X_{j}=k\right\} \\
&= \begin{cases}0 & \text { for } k=0 \\
1-\alpha & \text { for } k=M \\
(1-\alpha) \frac{k}{M} & \text { otherwise },\end{cases} \\
& q_{k, k}=\alpha,
\end{aligned}
$$

and

$$
\begin{aligned}
q_{k, k+1} & =P_{0}\left\{X_{j+1}=k+1 \mid X_{j}=k\right\} \\
& = \begin{cases}1-\alpha & \text { for } k=0 \\
0 & \text { for } k=M \\
1-\alpha-\frac{k(1-\alpha)}{M} & \text { otherwise. }\end{cases}
\end{aligned}
$$

In words, at the time of the $i$ th event, we pick a link uniformly at random out of all available links. With probability $\alpha$ nothing changes; otherwise in the event that a change will happen in the system, we delete or add a link in the following way: If the link was active (present) in the network, it is now deleted; otherwise it is now activated. Notice that the embedded dynamics are equivalent to the $\alpha$-delayed version of the Ehrenfest chain.

To connect the embedded chain $X_{n}$ with process $X(t)$, define the counting process

$$
N_{\beta}(t)=\max \left\{n \in \mathbb{N}: S_{n} \leqslant t\right\}
$$

that gives the number of events up to a finite time horizon $t$. This process is also called a fractional Poisson process. Then we have

$$
X(t)=X_{N_{\beta}(t)}=X_{n} \mathbb{1}\left\{S_{n} \leqslant t<S_{n+1}\right\},
$$

i.e., the state of the process at time $t$ is the same as that of the embedded chain after the last event before time $t$ occurred.

\section{B. Semi-Markov master equation}

All information about $X(t)$ is encoded in the pairs $\left\{\left(X_{n}, T_{n}\right)\right\}_{n \geqslant 1}$, which are a discrete-time Markov renewal 
process, satisfying

$$
\begin{aligned}
\mathbb{P}\left\{X_{n+1}\right. & \left.=j, T_{n+1} \leqslant t \mid\left(X_{0}, S_{0}\right), \ldots,\left(X_{n}=i, S_{n}\right)\right\} \\
& =\mathbb{P}\left\{X_{n+1}=j, T_{n+1} \leqslant t \mid X_{n}=i\right\} .
\end{aligned}
$$

Then $X(\cdot)$ is a semi-Markov process subordinated to $N_{\beta}(t)$ [18] and satisfies the forward equations

$p_{i, j}(t)=\bar{F}_{T}^{(\beta)}(t) \delta_{i j}+\sum_{\ell \in \mathcal{S}} q_{\ell, j} \int_{0}^{t} p_{i, \ell}(u) f_{T}^{(\beta)}(t-u) d u$.

Incidentally, a semi-Markov process is Markovian if and only if the distribution of $\left\{T_{n}\right\}_{n} \geqslant 1$ is exponential [27]. Above we introduced $p_{i, j}(t)=\mathbb{P}\{X(t)=j \mid X(0)=i\}$, the tail (complementary cumulative distribution function) $\bar{F}_{T}^{(\beta)}(t)=$ $1-F_{T}^{(\beta)}(t)$, and $f_{T}^{(\beta)}(t)$, the Mittag-Leffler density or order $\beta$. These equations are proved by conditioning on the time of the last event before time $t$. By taking Laplace transforms in (15) and using the known Laplace transform of the Mittag-Leffler survival function and probability density function

$$
\mathcal{L}\left(\bar{F}_{T}^{(\beta)}(t) ; s\right)=\frac{s^{\beta-1}}{1+s^{\beta}}, \quad \mathcal{L}\left(f_{T}^{(\beta)}(t) ; s\right)=\frac{1}{1+s^{\beta}},
$$

followed by some straightforward algebra, the evolution equations for $p_{i, j}(t)$ become (see the Appendix, Sec. 1)

$$
\begin{aligned}
\frac{d^{\beta} p_{i, j}(t)}{d t^{\beta}}= & -(1-\alpha) p_{i, j}(t)+(1-\alpha) \\
& \times\left(\frac{M-j+1}{M} p_{i, j-1}(t)+\frac{j+1}{M} p_{i, j+1}(t)\right) .
\end{aligned}
$$

Similarly, the equations of the boundary terms are

$$
\begin{gathered}
\frac{d^{\beta} p_{i, 0}(t)}{d t^{\beta}}=(1-\alpha)\left(-p_{i, 0}(t)+\frac{1}{M} p_{i, 1}(t)\right), \\
\frac{d^{\beta} p_{i, M}(t)}{d t^{\beta}}=(1-\alpha)\left(-p_{i, M}(t)+\frac{1}{M} p_{i, M-1}(t)\right) .
\end{gathered}
$$

The symbol $d^{\beta} / d t^{\beta}$ in (17)-(19) denotes the $\beta$ fractional Caputo derivative [22] of a function $f(t)$ given by

$$
\frac{d^{\beta} f(t)}{d t^{\beta}}=\frac{1}{\Gamma(1-\beta)} \int_{0}^{t}\left(t-t^{\prime}\right)^{-\beta} \frac{d f\left(t^{\prime}\right)}{d t^{\prime}} d t^{\prime} .
$$

When $\beta=1$, Eqs. (17)-(19) reduce (as expected) to the standard Kolmogorov equations for the Markovian RLAD [17]. These equations also explain analytically why $\alpha$ is called the delay parameter. When considering the scaled $\mathcal{D}_{\mathrm{ML}_{\gamma}}(\beta)$ times, Eq. (17) becomes

$$
\begin{aligned}
\frac{d^{\beta} p_{i, j}^{(\gamma)}(t)}{d t^{\beta}}= & -\gamma^{-\beta}(1-\alpha) p_{i, j}^{(\gamma)}(t)+\gamma^{-\beta}(1-\alpha) \\
& \times\left(\frac{M-j+1}{M} p_{i, j-1}^{(\gamma)}(t)+\frac{j+1}{M} p_{i, j+1}^{(\gamma)}(t)\right)
\end{aligned}
$$

and similarly for the boundary equations. Specifically we see, as in the Markovian case, that a scaled sojourn time distribution results in a (fractional) scalar multiple of the forward equations.

\section{Exact solution}

Equation (15) gives an analytical way to obtain the fractional equation for the evolution of the transition probabilities, but it is not very useful for computational purposes. Instead, it is fruitful to find the solution of the system of equations (17)-(19) by a simple conditioning argument on the values of $N_{\beta}(t)$ (see the Appendix, Sec. 2),

$$
p_{i, j}(t)=\bar{F}_{T}^{(\beta)}(t) \delta_{i j}+\sum_{n=1}^{\infty} q_{i, j}^{(n)} \mathbb{P}\left\{N_{\beta}(t)=n\right\},
$$

where $q_{i, j}^{(n)}$ are the $n$-step transitions of the embedded discrete Markov chain, namely, the entries of the $n$th power of the transition matrix $Q$ defined by Eqs. (9)-(11). The distribution of the fractional Poisson process has a simple expression generalizing the Poisson distribution [28], namely,

$$
\mathbb{P}\left\{N_{\beta}(t)=n\right\}=\frac{t^{\beta n}}{n !} E_{\beta}^{(n)}\left(-t^{\beta}\right),
$$

where $E_{\beta}^{(n)}\left(-t^{\beta}\right)$ denotes the $n$th derivative of $E_{\beta}(z)$ computed for $z=-t^{\beta}$. Equation (21) can also be verified to satisfy (17) using Laplace transforms (see the Appendix, Sec. 2).

\section{RESULTS AND APPLICATIONS}

All simulations are event driven, both for dynamic networks and when this is coupled with epidemic dynamics. Waiting times for all the possible events are generated from appropriate distributions. Hence, the next change or an update is always determined by the smallest waiting time and the event corresponding to it is executed. This is then followed by the necessary update of the waiting times of the events affected by the most recent change. In Ref. [29], readers can find an alternative efficient simulation method that effectively extends the ideas of the Gillespie algorithm from the Markovian to the non-Markovian case.

\section{A. Explicit calculation of $p_{i, j}(t)$}

The probabilities involving the counting process $N_{\beta}(t)$ have an explicit integral representation [30] and for numerical purposes they can be approximated well either with Monte Carlo simulations or with a numerical integration scheme. Once the transition probabilities of the embedded Markov chain are known, every term is known in (21) and it can be used to exactly calculate the nonequilibrium probabilities $p_{i, j}(t)$ (see the Appendix, Sec. 2). The excellent agreement between theory and simulation is shown in Fig. 1.

An immediate application is to use Eq. (21) and compute theoretically and numerically the expected number of active links in the network at a given time. Starting from any initial number of active links $i_{0}$, use (21) to compute

$$
\begin{aligned}
\mathbb{E}^{i_{0}}(X(t)) & =\mathbb{E}^{i_{0}}\left(\mathbf{e}_{i_{0}}^{T} Q^{N_{\beta}(t)} \mathbf{v}_{0, M}\right) \\
& =\mathbf{e}_{i_{0}}^{T} \mathbb{E}^{i_{0}}\left(Q^{N_{\beta}(t)}\right) \mathbf{v}_{0, M} .
\end{aligned}
$$

In the equation above $\mathbf{e}_{i_{0}}$ is the standard basis factor with 1 at the $i_{0}$ th coordinate and $\mathbf{v}_{0, M}=(0,1,2, \ldots, M)^{T}$. Note that in the particular case where $Q$ diagonalizes, the analytical expression for the expectation (23) is merely a linear 




FIG. 1. (Color online) Comparison between Monte Carlo simulations and theory. The discrete markers are the estimated probabilities $p_{190, j}(250)$, averaged over 10000 Monte Carlo simulations starting from a fully connected network with $N=20$ nodes and for $\beta=1,0.7,0.5$, as we move from left to right. The Monte Carlo simulations were performed using an event-driven algorithm taking non-Markovianity into account. The solid curves are the theoretical predictions as dictated by Eq. (21). combination of different values of the probability generating function of $N_{\beta}(t), G^{(\beta)}(s ; t)$, given by (see [31]) $G^{(\beta)}(z ; t)=$ $\mathbb{E}\left(z^{N_{\beta}(t)}\right)=E_{\beta}\left((z-1) t^{\beta}\right)$. Particularly, when $Q$ diagonalizes, there is no need for simulating a large number of realizations to estimate the expectation; a fast numerical integration scheme is sufficient.

\section{B. Approximation of Pareto-distributed interevent times}

We now compare the behavior between two RLAD networks: one with Mittag-Leffler times and one where we alter the waiting-time distribution to a generalized $\mathcal{D}_{\mathrm{P}}(\delta)$ with density

$$
f_{T}(t)=\frac{\delta-1}{(1+t)^{\delta}}, \quad t>0 .
$$

The exponent $\delta=1+\beta$ in order for the tails of Mittag-Leffler and the Pareto distributions to have the same behavior at infinity. In fact, we compare the two networks over three layers of increasing complexity. First, in Figs. 2(b) and 2(c) we plot the probability mass function for the number of singly counted links at a prespecified time horizon $T=2000$, averaged over 5000 simulations. As a point of reference, output from the Markovian RLAD is shown in Fig. 2(a). The theoretical curve at equilibrium is the large time limit of Eq. (21) and it is the mass function of a binomial distribution with $M$ trials and
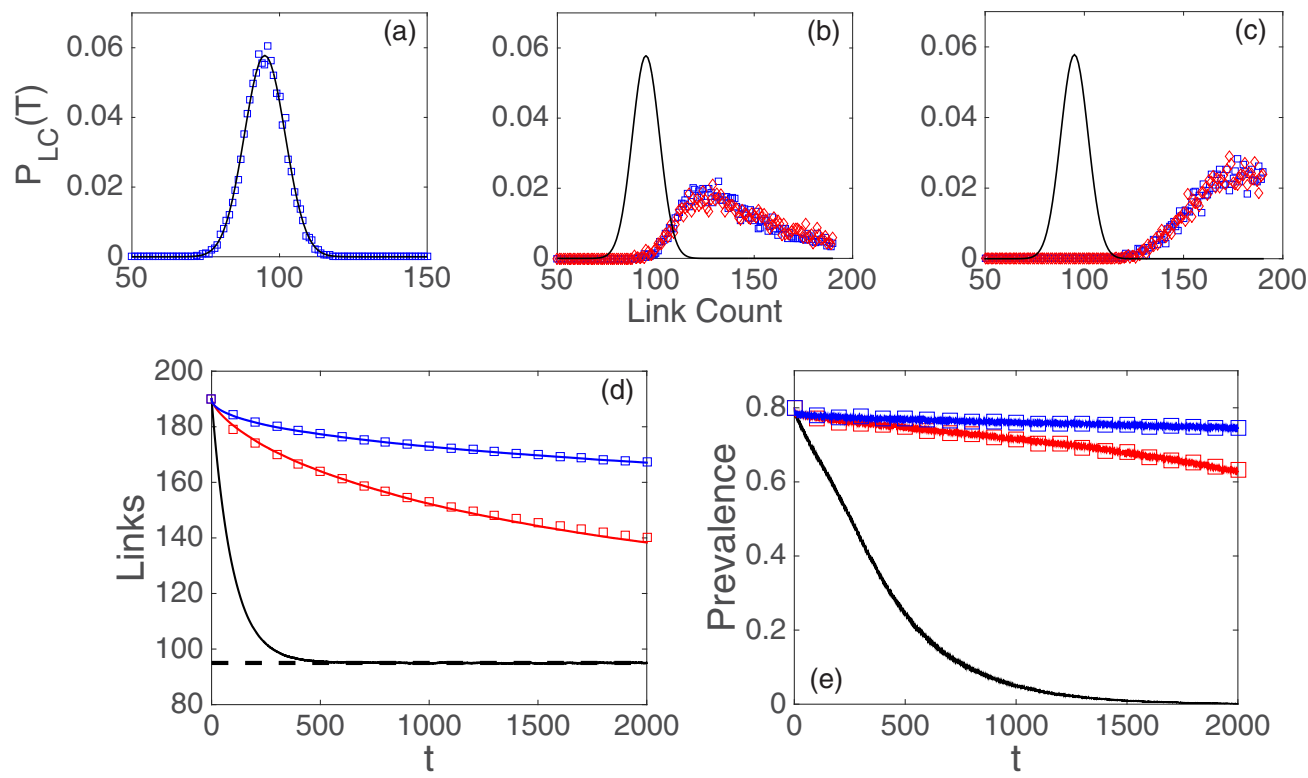

FIG. 2. (Color online) (a)-(c) Distribution of links at $t=2000$ and comparison between the Mittag-Leffler and Pareto distributions. The distribution of singly counted link numbers at $t=2000$ is shown with theoretical equilibrium prediction (solid line) and simulation results (discrete markers). The theoretical equilibrium is the same for all values of $\beta \in(0,1]$ and is given by the binomial distribution (25) here drawn as a solid line for ease of representation. The $\square$ markers in (a), (b), and (c) correspond to $(\beta, \gamma)=(1,1),(0.7,3.14),(0.5,4)$, respectively, and $\diamond$ markers are for the corresponding $\mathcal{D}_{\mathrm{P}}(\delta)$. Also shown are (d) the expected number of singly counted links in the network [the dashed line is the theoretical prediction of $N(N-1) / 4=95]$ and (e) the expected prevalence. The solid (noisy) line is for $(\beta, \gamma)=(1,1),(0.7,3.14),(0.5,4)$, respectively, from bottom to top and $\square$ markers are the corresponding values for the matched Pareto network. The networks have $N=20$ nodes and simulations start with a completely connected network. The SIS epidemic is simulated as a Markovian process with transmission and recovery rates $\tau_{I}=0.25$ and $\tau_{H}=1$, respectively. The spreading process initializes with five infectious nodes. Since we start with a fully connected network and a slow network dynamics, the prevalence rapidly increases from $25 \%$ to almost $80 \%$. This also reflects the relation between the time scales of the network and epidemic dynamics, with the epidemics being much faster in this example. The simulation is event driven and is implemented by keeping track of all events and their waiting times. These are averaged over 5000 simulations and use $\alpha=0$ (periodic case), so all events create a change in the network and equilibrium is reached earlier. 
success probability $1 / 2$. Second, in Fig. 2(d) we plot $\mathbb{E}(X(t))$ as a function of time up to time $T=2000$. The two curves could also be computed based on Eq. (23).

The excellent agreement is achieved by finding a suitable scaling $\gamma$ so that the complementary CDF (CCDF) (survival functions) of the two distributions match well, at least up to the prespecified time horizon that we want to study. Further details can be found in the Appendix, Sec. 3. The matching is good for $\beta<0.9$ while for larger $\beta$, this idea can be used to study the stochastic dominance between the two coupled networks and offer rigorous bounds.

\section{Markovian SIS on non-Markovian RLAD}

Finally, we compare the two network dynamics indirectly, when we allow a Markovian epidemic to run while the networks evolve. As discussed above, human activity tends to be bursty and non-Markovian [32]. During an epidemic, individuals become wary of the risk posed by it and one way to avoid infection is by limiting or reducing their number of contacts. This justifies the deletion of links as time evolves. On the other hand, close contacts cannot realistically be removed and some level of communication and social cohesion must be maintained. Such behavior in activation and deletion is not necessarily Markovian in nature, thus alternative nonMarkovian dynamic network models are necessary.

Nodes in the network represent individuals from a population and links describe the contact patterns among these. Each individual can be either infected $I$ or susceptible $S$. An infected individual remains infected for exponentially distributed periods of time $T_{H}$, i.e., $T_{H} \sim \mathcal{E}\left(1 / \tau_{H}\right)$, where $\tau_{H}$ is the average time in which infectious individuals are healed. Similarly, infection occurs at the points of a Poisson process with time to infection $T_{I}$ exponentially distributed, i.e., $T_{I} \sim \mathcal{E}\left(1 / \tau_{I}\right)$, where $\tau_{I}$ is the average time in which an infection spreads across a link connecting a susceptible and an infected node. In this framework, both network and epidemic dynamics can be considered in the context of event-driven simulations, where the timing of the next state change is always determined by the smallest waiting time and the precise event corresponding to it. The epidemic does not interfere with the network dynamics, however its propagation is intertwined with the background dynamic network topology. Initially, before the infection starts spreading, we assume that all links are present, in order to avoid early stochastic extinction.

The simulations in Fig. 2 show the prevalence (proportion of infected individuals [Fig. 2(e)]) on a $\mathcal{D}_{\mathrm{ML}_{\gamma}}(\beta)$ RLAD network (solid lines) and a direct comparison (square markers) with the $\mathcal{D}_{\mathrm{P}}(\delta)$. Again, we use the same sets of $\beta, \gamma, \delta$ as before and we emphasize the excellent agreement between the two.

Incidentally, as expected, the non-Markovian network dynamics create a striking effect by slowing down the network dynamics and thus effectively blocking the attainment of statistical equilibrium in a realistic time horizon [Figs. 1 and 2(a)-2(c)]. This leads to a heightened level of infectiousness in the population [Fig. 2(e)] and highlights the importance of quick reactions. Naturally the statistical equilibrium will be reached after a much longer time period, but the delayed curves can now be theoretically computed or approximated.
At equilibrium the number of active links is a continuoustime irreducible birth-death chain with a unique binomial invariant distribution $\pi$, independent of the delay parameter $\alpha$, given by

$$
\pi_{k}=\lim _{t \rightarrow \infty} \mathbb{P}\{X(t)=k \mid X(0)=i\}=\left(\begin{array}{c}
M \\
k
\end{array}\right) 2^{-M}, \quad k \in \mathcal{S} .
$$

This can also be deduced from the fact that in the aperiodic $\alpha$ delayed case, at equilibrium, individual graphs are uniformly distributed. That is because the chain on the set of distinct graphs has a doubly stochastic transition matrix. With this in mind, the degree distribution of a single node in network chosen uniformly at random can be immediately computed as follows. Let $v_{1}$ be a selected node in $G$ and define $G_{v_{1}}$ the subgraph of $G$ where $v_{1}$ and all its incident links are deleted. Now $G_{v_{1}}$ is a graph on the set $\left\{v_{2}, \ldots, v_{N}\right\}$ that has at most $K=$ $\left(\begin{array}{c}N-1 \\ 2\end{array}\right)=M-N+1$ links. Let $\operatorname{deg}\left(v_{1}\right)$ denote the degree of $v_{1}$. Then

$$
\begin{aligned}
\mathbb{P}\left\{\operatorname{deg}\left(v_{1}\right)=\ell\right\} & =\sum_{\operatorname{graphs} G: \operatorname{deg}\left(v_{1}\right)=\ell} 2^{-M} \\
& =2^{-M} \operatorname{card}\left\{\operatorname{graphs} G: \operatorname{deg}\left(v_{1}\right)=\ell\right\} \\
& =2^{-M}\left(\begin{array}{c}
N-1 \\
\ell
\end{array}\right) \operatorname{card}\left\{\operatorname{graphs} G_{v_{1}}\right\} \\
& =2^{-K-N+1}\left(\begin{array}{c}
N-1 \\
\ell
\end{array}\right) \sum_{i=0}^{K}\left(\begin{array}{c}
K \\
i
\end{array}\right) \\
& =\left(\begin{array}{c}
N-1 \\
\ell
\end{array}\right) 2^{-N+1} .
\end{aligned}
$$

The third equality is a counting argument. The number of graphs such that $v_{1}$ has exactly $\ell$ incident links is constructed by first selecting where those links go and then constructing the subgraph $G_{v_{1}}$. Equation (26) can be understood heuristically as follows. Select a link and focus on one of the two nodes. If this node has $h$ active links, this number will go either to $h+1$ with probability $(N-1-h) /(N-1)$ or to $h-1$ with probability $h /(N-1)$. This leads to an invariant binomial degree distribution (26), with an average degree of $(N-1) / 2$, which amounts to $N(N-1) / 4$ active edges in the network in line with the average of the link distribution from (25).

Finally we discuss the delayed convergence to equilibrium, via the embedded chain mixing times, in the total variation distance. The chain mixing time $t_{\text {mix }}(\varepsilon)$ is the minimal time so the total variation distance between the measures $\pi$ and $p(t)$ is smaller than some tolerance $\varepsilon$, i.e.,

$$
\left\|p\left(t_{\text {mix }}(\varepsilon)\right)-\pi\right\|_{\mathrm{TV}}=\sup _{k \in \mathcal{S}}\left|p_{k}\left(t_{\text {mix }}(\varepsilon)\right)-\pi_{k}\right|<\varepsilon .
$$

For the Markovian RLAD with $\alpha>0$, use Theorem 1.1 and Example 4.3 in [33] to see

$$
t_{\text {mix }}(\varepsilon) \leqslant C \varepsilon^{-2} M^{2} \ln M .
$$

Thus, the Markov chain approximates relatively well its equilibrium by time $C \varepsilon^{-2} M^{2} \ln M$. In particular this implies that, on average, the Markovian RLAD continuous chain needs $O\left(M^{2} \ln M\right)$ time and therefore by the law of large numbers, it needs this order many events until it is well mixed. In fact, 
this bound is also true for the embedded discrete chain. This $n=M^{2} \ln M$ should be considered as a necessary lower bound of steps so that the sample average of the probabilities of the embedded chain approximates $\pi$. Therefore, in order to have an acceptable level of accuracy for the embedded chain when the RLAD has Mittag-Leffler waiting times, using (22), we need a higher-polynomial-order $O\left(M^{2 / \beta}(\ln M)^{1 / \beta}\right)$ time in order to guarantee on average the same number of events and thus to guarantee a near equilibrium behavior for the embedded chain. A study of the slowdown phenomenon for non-Markovian dynamic networks, using the total variation distance, can be found, for example, in [34].

\section{DISCUSSION AND CONCLUSION}

\section{A. Generalization}

Equation (21) can be generalized to any counting process and any discrete Markov chain and, as a consequence, to any embedded Markovian graph dynamics [18]. To be more specific, let $q_{i, j}$ denote the one-step transition probability from state $i$ to state $j$ for a discrete Markov chain $X_{n}$ and let $N(t)$ be a generic counting renewal process. Then, for the process

$$
X(t)=X_{N(t)}=X_{n} \mathbb{1}\left\{S_{n} \leqslant t<S_{n+1}\right\},
$$

the probabilities $p_{i, j}(t)=\mathbb{P}\{X(t)=j \mid X(0)=i\}$ are given by

$$
p_{i, j}(t)=\bar{F}_{T}(t) \delta_{i j}+\sum_{n=1}^{\infty} q_{i, j}^{(n)} \mathbb{P}\{N(t)=n\},
$$

where the symbols have the same meaning as in (21) and $\left\{T_{i}\right\}_{i=1}^{\infty}$ is a sequence of independent and identically distributed positive random variables with the usual meaning of interevent times with arbitrary distribution, not necessarily with fat tails and infinite mean. The reader is invited to follow the first proof of the Appendix, Sec. 2 by replacing $N_{\beta}(t)$ with a generic counting renewal process. This will convince the reader of the wide generality of this result. A heuristic argument to justify (21) and (30) runs as follows. In the time interval $(0, t), n \geqslant 0$ events may have occurred. In the case $n=0$, at time $t$ the process is still in state $i$ and $\mathbb{P}\{N(t)=0\}=$ $\mathbb{P}\{T>t\}=\bar{F}_{T}(t)$. If $n \geqslant 1$, the probability of being in state $j$ after $n$ events is given by $q_{i, j}^{(n)}$. Given the independence between the renewal process and the Markov chain, the probability of being in state $j$ at time $t$ and $n$ transitions occurring in the time interval $(0, t)$ is $q_{i, j}^{(n)} \mathbb{P}\{N(t)=n\}$. All these events are exhaustive and mutually exclusive. Then the total probability and infinite additivity imply that $p_{i, j}(t)$ is given by (30). These considerations suggest further generalizations taking into account a possible dependence within the couple $\left\{\left(X_{n}, T_{n}\right)\right\}_{n \geqslant 1}$ as well as a serial dependence or state dependence of interevent times.

\section{B. Example: Simple probabilistic model for relaxation in dielectrics}

In order to illustrate the generalization discussed above with an example, we consider relaxation phenomena [35]. Probabilistic modeling of relaxation assumes that a physical system (e.g., a molecule) can exist in two states $A$ and $B$. We further assume that state $A$ is transient and state $B$ absorbing, so that the deterministic embedded chain has the transition probabilities $q_{A, A}=0, q_{A, B}=1, q_{B, A}=0$, and $q_{B, B}=1$. This means that if the system is prepared in state $A$, it will jump to state $B$ at the first step and it will stay there forever. Now suppose that the interevent time $T$ is random and follows an exponential distribution with rate $\lambda=1$ for the sake of simplicity. Based on Eq. (30), we immediately have $p_{A, A}(t)=\bar{F}_{T}(t)=\exp (-t)$. Therefore, the probability of finding the system in the initial state decays exponentially towards zero. This relaxation function is the solution of

$$
\frac{d}{d t} p_{A, A}(t)=-p_{A, A}(t), \quad p_{A, A}(0)=1 .
$$

The response function is defined as $\xi_{D}(t)=-d p_{A, A}(t) / d t$ and its Laplace transform is $1 /(1+s)$. For $s=-i \omega$ this is the Debye model [35]. If interevent times follow the Mittag-Leffler distribution, we get $p_{A, A}(t)=\bar{F}_{T}(t)=E_{\beta}\left(-t^{\beta}\right)$. This is the solution of [28]

$$
\frac{d^{\beta}}{d t^{\beta}} p_{A, A}(t)=-p_{A, A}(t), \quad p_{A, A}(0)=1 .
$$

In this case, the Laplace transform of the response function $\xi_{C C}(t)=-d p_{A, A}(t) / d t$ is $1 /\left(1+s^{\beta}\right)$ and for $s=-i \omega$, we get the Cole-Cole model [35-37].

\section{Final considerations}

In conclusion, we have provided an exactly solvable nonMarkovian dynamic network model. The RLAD is particularly attractive as it has analytical and numerical tractability coming from fractional calculus. We were able to explicitly use the master equation formalism and analytically derive the distribution of the number of links in the network for arbitrary times $X(t)$, consequently computing $\mathbb{E}(X(t))$. We highlighted an important connection and possible avenue to approximate non-Markovian problems using fractional calculus, by coupling a Pareto network, and showed the agreement with the tractable model. Moreover, we discussed how our result can be extended to a generic counting renewal process.

\section{ACKNOWLEDGMENTS}

N. Georgiou and E. Scalas were partially supported by the Strategic Development Fund (SDF) provided by the University of Sussex.

\section{APPENDIX}

In this appendix we cover the rigorous proofs of the equations shown in the main text and further clarify some notions. Some details about the procedure used to couple the Pareto distribution with the Mittag-Leffler are also highlighted.

\section{Derivation of fractional equations}

We want to show that Eqs. (17)-(19) are obtained from (15). The analysis proceeds by way of Laplace transforms. They are defined as

$$
\mathcal{L}(g(t) ; s)=\int_{0}^{\infty} d t g(t) e^{-s t}
$$


for a suitable function $g(t)$. In the case of the Mittag-Leffler distribution defined in the main text, we have

$$
\mathcal{L}\left(\bar{F}_{T}^{(\beta)}(t) ; s\right)=\frac{s^{\beta-1}}{1+s^{\beta}} \mathcal{L}\left(f_{T}^{(\beta)}(t) ; s\right)=\frac{1}{1+s^{\beta}} .
$$

For the computation that follows we use the symbol $\tilde{g}(s)$ to denote the Laplace transform $\mathcal{L}(g ; s)$ of any function $g$. Taking the Laplace transform of (15) and using Eqs. (4)-(6) for our particular example, we have, for $1 \leqslant j \leqslant M-1$,

$$
\begin{aligned}
\tilde{p}_{i, j}(s)= & \widetilde{\bar{F}}_{T}^{(\beta)}(s) \delta_{i j}+\tilde{f}_{T}^{(\beta)}(s) \alpha \tilde{p}_{i, j}(s)+\tilde{f}_{T}^{(\beta)}(s)(1-\alpha) \\
& \times\left(\frac{M-j+1}{M} \tilde{p}_{i, j-1}(s)+\frac{j+1}{M} \tilde{p}_{i, j+1}(s)\right) .
\end{aligned}
$$

The boundary cases $j=0$ and $j=M$ have Laplace transforms

$$
\begin{aligned}
\tilde{p}_{i, 0}(s)= & \widetilde{\widetilde{F}}_{T}^{(\beta)}(s) \delta_{i 0}+\tilde{f}_{T}^{(\beta)}(s)\left(\frac{1-\alpha}{M} \tilde{p}_{i, 1}(s)+\alpha \tilde{p}_{i, 0}(s)\right), \\
\tilde{p}_{i, M}(s)= & \widetilde{F}_{T}^{(\beta)}(s) \delta_{i M} \\
& \left.+\tilde{f}_{T}^{(\beta)}(s)\left(\frac{1-\alpha}{M} \tilde{p}_{i, M-1}(s)+\alpha \tilde{p}_{i, M}(s)\right), \quad \text { (A } 5\right)
\end{aligned}
$$

respectively. We finish the computation starting from (A3), in the case where $1 \leqslant j \leqslant n-1$. The remaining cases follow similarly. Multiply both sides of (A3) by $s$ and then subtract $p_{i, j}(0)=\delta_{i j}$ from both sides. Then, using (A2), Eq. (A3) becomes

$$
\begin{aligned}
\mathcal{L}( & \left.\frac{d p_{i, j}(t)}{d t} ; s\right) \\
= & s \widetilde{\bar{F}}_{T}^{(\beta)}(s) \delta_{i j}-p_{i, j}(0)+\frac{s}{1+s^{\beta}} \alpha \tilde{p}_{i, j}(s) \\
& \quad+\frac{s(1-\alpha)}{1+s^{\beta}}\left(\frac{M-j+1}{M} \tilde{p}_{i, j-1}(s)+\frac{j+1}{M} \tilde{p}_{i, j+1}(s)\right) \\
= & \frac{s^{\beta}}{1+s^{\beta}} \delta_{i j}-\delta_{i j}+\frac{s}{1+s^{\beta}} \alpha \tilde{p}_{i, j}(s) \\
& +\frac{s(1-\alpha)}{1+s^{\beta}}\left(\frac{M-j+1}{M} \tilde{p}_{i, j-1}(s)+\frac{j+1}{M} \tilde{p}_{i, j+1}(s)\right),
\end{aligned}
$$

thus, after some algebraic manipulations we have

$$
\begin{aligned}
& \frac{1+s^{\beta}}{s} \mathcal{L}\left(\frac{d p_{i, j}(t)}{d t} ; s\right) \\
& =\frac{-\delta_{i j}}{s}+\alpha \tilde{p}_{i, j}(s) \\
& \quad+(1-\alpha)\left(\frac{M-j+1}{M} \tilde{p}_{i, j-1}(s)+\frac{j+1}{M} \tilde{p}_{i, j+1}(s)\right)
\end{aligned}
$$

Focus for the moment on the factor $s^{-1}\left(1+s^{\beta}\right)$. Its inverse Laplace transform is

$$
\mathcal{L}^{-1}\left(s^{-1}\left(1+s^{\beta}\right) ; t\right)=\frac{t^{-\beta}}{\Gamma(1-\beta)}+1=\Phi_{\beta}(t)+1 .
$$

The kernel $\Phi_{\beta}(t)$ is what is used in fractional calculus to define the $\beta$ fractional Caputo derivative (see Ref. [19]) of a function $f(t)$, given by

$$
\frac{d^{\beta} f(t)}{d t^{\beta}}=\int_{0}^{t} \Phi_{\beta}\left(t-t^{\prime}\right) \frac{d f\left(t^{\prime}\right)}{d t^{\prime}} d t^{\prime} .
$$

Thus, use (A7) to write the left-hand side of (A6) as a product of two Laplace transforms. Then take the Laplace inverse of (A6) to conclude

$$
\begin{aligned}
\frac{d^{\beta} p_{i, j}(t)}{d t^{\beta}}= & -(1-\alpha) p_{i, j}(t)+(1-\alpha) \\
& \times\left(\frac{M-j+1}{M} p_{i, j-1}(t)+\frac{j+1}{M} p_{i, j+1}(t)\right) .
\end{aligned}
$$

Similarly, the equations of the boundary terms are derived (18) and (19).

\section{Solution to the fractional equations}

The solution to Eqs. (12)-(14) can be seen to be Eq. (15) in two different ways. One is the standard law of total probability, where the space is partitioned according to the number of jumps of the counting process $N_{\beta}(t)$ :

$$
\begin{aligned}
p_{i, j}(t)= & \mathbb{P}\{X(t)=j \mid X(0)=i\} \\
= & \sum_{k=0}^{\infty} \mathbb{P}\left\{X(t)=j, N_{\beta}(t)=k \mid X(0)=i\right\} \\
= & \sum_{k=0}^{\infty} \mathbb{P}\left\{X(t)=j \mid N_{\beta}(t)=k, X(0)=i\right\} \mathbb{P}\left\{N_{\beta}(t)=k\right\} \\
= & \mathbb{P}\left\{X(t)=j \mid N_{\beta}(t)=0, X(0)=i\right\} \mathbb{P}\left\{N_{\beta}(t)=0\right\} \\
& +\sum_{k=1}^{\infty} \mathbb{P}\left\{X(t)=j \mid N_{\beta}(t)=k, X(0)=i\right\} \\
& \times \mathbb{P}\left\{N_{\beta}(t)=k\right\} \\
= & \mathbb{P}\left\{X(t)=j \mid T_{1} \geqslant t, X(0)=i\right\} \mathbb{P}\left\{T_{1} \geqslant t\right\} \\
& +\sum_{k=1}^{\infty} \mathbb{P}\left\{X(t)=j \mid N_{\beta}(t)=k, X(0)=i\right\} \\
& \times \mathbb{P}\left\{N_{\beta}(t)=k\right\} \\
= & \delta_{i j} \bar{F}_{T}^{(\beta)}(t) \\
& +\sum_{k=1}^{\infty} \mathbb{P}\left\{X_{k}=j \mid \mathbb{1}\left\{S_{k} \leqslant t<S_{k+1}\right\}, X_{0}=i\right\} \\
& \times \mathbb{P}_{i j} \bar{F}_{T}^{(\beta)}(t)+\sum_{k=1}^{\infty} \mathbb{P}\left\{X_{k}=j \mid X_{0}=i\right\} \mathbb{P}\left\{N_{\beta}(t)=k\right\} \\
& \\
& \\
&
\end{aligned}
$$

where it finally leads to

$$
p_{i, j}(t)=\delta_{i j} \bar{F}_{T}^{(\beta)}(t)+\sum_{k=1}^{\infty} q_{i j}^{(k)} \mathbb{P}\left\{N_{\beta}(t)=k\right\} .
$$


Equation (A9) is Eq. (21) and as we say gives the theoretical solution to the fractional equations because of an explicit integral representation of $\mathbb{P}\left\{N_{\beta}(t)=n\right\}$. It is given by

$$
\begin{aligned}
\mathbb{P}\left\{N_{\beta}(t)=n\right\} & =\frac{t^{\beta n}}{n !} E_{\beta}^{(n)}\left(-t^{\beta}\right) \\
& =\int_{0}^{\infty} F_{S_{\beta}}(t ; u)\left(1-\frac{u}{n}\right) \frac{u^{n-1}}{(n-1) !} e^{-u} d u .
\end{aligned}
$$

The function $F_{S_{\beta}}(t ; u)$ is the $\mathrm{CDF}$ of a stable random variable $S_{\beta}(v, \gamma, \delta)$ with index $\beta$, skewness parameter $v=1$, scale $\gamma=[u \cos (\pi \beta / 2)]^{1 / \beta}$, and location $\delta=0$. This integral representation was used to numerically compute the solid curve in Fig. 1 [30].

We now verify via Laplace transforms that this solution (A9) indeed verifies the fractional equations. For simplicity we set the delay parameter $\alpha=0$ and we only show it for Eq. (17). We need

$$
\begin{aligned}
& \mathcal{L}\left(\frac{d^{\beta} g}{d t^{\beta}} ; s\right)=s^{\beta} \tilde{g}(s)-s^{\beta-1} g(0+), \\
& \mathcal{L}\left(\mathbb{P}\left\{N_{\beta}(t)=n\right\} ; s\right)=\widetilde{\widetilde{F}}_{T}^{(\beta)}(s)\left[\tilde{f}_{T}^{(\beta)}(s)\right]^{n}=\frac{\widetilde{\bar{F}}_{T}^{(\beta)}(s)}{\left(1+s^{\beta}\right)^{n}} .
\end{aligned}
$$

The Laplace transform of (17)

$$
\begin{aligned}
s^{\beta} \tilde{p}_{i, j}(s)-s^{\beta-1} \delta_{i j}= & -\tilde{p}_{i, j}(s)+\frac{M-j+1}{M} \tilde{p}_{i, j-1}(s) \\
& +\frac{j+1}{M} \tilde{p}_{i, j+1}(s),
\end{aligned}
$$

or after an algebraic manipulation

$$
\left(1+s^{\beta}\right) \tilde{p}_{i, j}(s)=s^{\beta-1} \delta_{i j}+q_{j-1, j} \tilde{p}_{i, j-1}(s)+q_{j+1, j} \tilde{p}_{i, j+1}(s) .
$$

To verify (A10), directly take the Laplace transform in (A9) to write

$$
\tilde{p}_{i, j}(s)=\delta_{i j} \widetilde{\widetilde{F}}_{T}^{(\beta)}(s)+\widetilde{\widetilde{F}}_{T}^{(\beta)}(s) \sum_{k=1}^{\infty} q_{i j}^{(k)}\left[\tilde{f}_{T}^{(\beta)}(s)\right]^{k}
$$

and substitute into the right-hand side in (A10), which now reads

$$
\begin{aligned}
& s^{\beta-1} \delta_{i j}+q_{j-1, j} \tilde{p}_{i, j-1}(s)+q_{j+1, j} \tilde{p}_{i, j+1}(s) \\
& =s^{\beta-1} \delta_{i j}+q_{j-1, j} \widetilde{\bar{F}}_{T}^{(\beta)}(s)\left(\delta_{i, j-1}+\sum_{k=1}^{\infty} q_{i, j-1}^{(k)}\left[\tilde{f}_{T}^{(\beta)}(s)\right]^{k}\right) \\
& \quad+q_{j+1, j} \widetilde{\widetilde{F}}_{T}^{(\beta)}(s)\left(\delta_{i, j+1}+\sum_{k=1}^{\infty} q_{i, j+1}^{(k)}\left[\tilde{f}_{T}^{(\beta)}(s)\right]^{k}\right) \\
& =s^{\beta-1} \delta_{i j}+\left(q_{j-1, j} \delta_{i, j-1}+q_{j+1, j} \delta_{i, j+1}\right) \widetilde{\bar{F}}_{T}^{(\beta)}(s) \\
& \quad+\widetilde{\widetilde{F}}_{T}^{(\beta)}(s) \sum_{k=1}^{\infty}\left(q_{j-1, j} q_{i, j-1}^{(k)}+q_{j+1, j} q_{i, j+1}^{(k)}\right)\left[\tilde{f}_{T}^{(\beta)}(s)\right]^{k} \\
& =s^{\beta-1} \delta_{i j}+q_{i, j} \widetilde{\bar{F}}_{T}^{(\beta)}(s) \\
& \quad+\left(1+s^{\beta}\right) \widetilde{\bar{F}}_{T}^{(\beta)}(s) \sum_{k=1}^{\infty} q_{i, j}^{(k+1)}\left[\tilde{f}_{T}^{(\beta)}(s)\right]^{k+1}
\end{aligned}
$$

$$
\begin{aligned}
= & s^{\beta-1} \delta_{i j}+q_{i, j} s^{\beta-1} \tilde{f}_{T}^{(\beta)}(s) \\
& +\left(1+s^{\beta}\right) \widetilde{\bar{F}}_{T}^{(\beta)}(s) \sum_{k=1}^{\infty} q_{i, j}^{(k+1)}\left[\tilde{f}_{T}^{(\beta)}(s)\right]^{k+1} \\
= & \left(1+s^{\beta}\right) \widetilde{\bar{F}}_{T}^{(\beta)}(s)\left[\delta_{i j}+\sum_{k=1}^{\infty} q_{i, j}^{(k)}\left[\tilde{f}_{T}^{(\beta)}(s)\right]^{k}\right] \\
= & \left(1+s^{\beta}\right) \tilde{p}_{i, j}(s),
\end{aligned}
$$

which is the left-hand side of (A10).

\section{Stochastic coupling with the Pareto distribution}

Now we show how the complementary cumulative distribution functions of the $\mathcal{D}_{\mathrm{P}}(\delta)$ distribution and the $\mathcal{D}_{\mathrm{ML}_{\gamma}}(\beta)$ of the same exponent can be shown to match just by manipulating the scaling factor $\gamma$. On a double logarithmic scale the CCDFs have a linear behavior at infinity with slope $1-\delta=-\beta$. Our simulations have a time horizon of $T=2000$, so the scaling $\gamma$ is chosen so that the CCDFs agree well for values around and before the time horizon. The initial value of $\gamma$ to be tested for matching is the solution to the equation

$$
\frac{\sin (\beta \pi)}{\pi} \frac{\Gamma(\beta)}{(t / \gamma)^{\beta}}=\frac{1}{t^{\delta-1}} \Longleftrightarrow \gamma=\left(\frac{\pi}{\sin (\beta \pi) \Gamma(\beta)}\right)^{1 / \beta},
$$

which implies the agreement of the asymptotic behavior of the survival functions. This first $\gamma$ choice will need to be adjusted, depending on our choice of time horizon, but the match can be achieved relatively well for moderate $\beta$ values $(\beta<0.9)$ (see Fig. 3).


FIG. 3. (Color online) The CCDF of a Pareto distribution with tail exponent $\delta$ and that matching with the corresponding $\mathcal{D}_{\mathrm{ML}_{\gamma}}(\beta)$. The left panel presents the case $\beta=0.7, \gamma=4$, and $\delta=1.7$, whereas the right panel presents the case $\beta=0.5, \gamma=3.14$, and $\delta=1.5$. The Pareto distribution is drawn using diamonds, whereas the MittagLeffler distribution is drawn using a solid line. 
[1] A. L. Lloyd, Theor. Popul. Biol. 60, 59 (2001).

[2] E. Scalas, T. Kaizoji, M. Kirchler, J. Huber, and A. Tedeschi, Physica A 366, 463 (2006).

[3] J.-P. Eckmann, E. Moses, and D. Sergi, Proc. Natl. Acad. Sci. USA 101, 14333 (2004).

[4] R. D. Malmgren, D. B. Stouffer, A. E. Motter, and L. A. Amaral, Proc. Natl. Acad. Sci. USA 105, 18153 (2008).

[5] Z.-Q. Jiang, W.-J. Xie, M.-X. Li, B. Podobnik, W.-X. Zhou, and H. E. Stanley, Proc. Natl. Acad. Sci. USA 110, 1600 (2013).

[6] C. M. Schneider, V. Belik, T. Couronné, Z. Smoreda, and M. C. González, J. R. Soc. Interface 10, 20130246 (2013).

[7] A. Moinet, M. Starnini, and R. Pastor-Satorras, Phys. Rev. Lett. 114, 108701 (2015).

[8] T. Hoffmann, M. A. Porter, and R. Lambiotte, Phys. Rev. E 86, 046102 (2012).

[9] B. Min, K.-I. Goh, and A. Vazquez, Phys. Rev. E 83, 036102 (2011).

[10] P. Van Mieghem and R. van de Bovenkamp, Phys. Rev. Lett. 110, 108701 (2013).

[11] H.-H. Jo, J. I. Perotti, K. Kaski, and J. Kertész, Phys. Rev. X 4, 011041 (2014).

[12] P. Holme and J. Saramäki, Phys. Rep. 519, 97 (2012).

[13] N. Perra, B. Gonçalves, R. Pastor-Satorras, and A. Vespignani, Sci. Rep. 2, 469 (2012).

[14] S. Vajna, B. Tóth, and J. Kertész, New J. Phys. 15, 103023 (2013).

[15] T. Gross and H. Sayama, Adaptive Networks (Springer, Berlin, 2009).

[16] V. Marceau, P.-A. Noël, L. Hébert-Dufresne, A. Allard, and L. J. Dubé, Phys. Rev. E 82, 036116 (2010).

[17] I. Z. Kiss, L. Berthouze, T. J. Taylor, and P. L. Simon, Proc. R. Soc. London Ser. A 468, 1332 (2012).

[18] M. Raberto, F. Rapallo, and E. Scalas, PLoS ONE 6, e23370 (2011).
[19] P. Hänggi and P. Talkner, Z. Phys. B 45, 79 (1981).

[20] E. Orsingher and F. Polito, Bernoulli 17, 114 (2011).

[21] M. M. Meerschaert and B. Toaldo, arXiv:1506.02951.

[22] D. Baleanu, K. Diethelm, E. Scalas, and J. J. Trujillo, Fractional Calculus: Models and Numerical Methods (World Scientific, Singapore, 2012).

[23] F. Mainardi and R. Gorenflo, in Fractals and Fractional Calculus in Continuum Mechanics, edited by A. Carpinteri and F. Mainardi (Springer, Berlin, 1997), pp. 223-276.

[24] S. Bernstein, Acta Math. 52, 1 (1928).

[25] R. Schilling, R. Song, and Z. Vondraček, Bernstein Functions: Theory and Applications (De Gruyter, Boston, 2010).

[26] H. Pollard, Bull. Am. Math. Soc. 54, 1115 (1948).

[27] E. Çinlar, Introduction to Stochastic Processes (Dover, New York, 2013).

[28] E. Scalas, R. Gorenflo, and F. Mainardi, Phys. Rev. E 69, 011107 (2004).

[29] M. Boguñá, L. F. Lafuerza, R. Toral, and M. A. Serrano, Phys. Rev. E 90, 042108 (2014).

[30] M. Politi, T. Kaizoji, and E. Scalas, Europhys. Lett. 96, 20004 (2011).

[31] N. Laskin, Commun. Nonlinear Sci. Numer. Simul. 8, 201 (2003).

[32] A.-L. Barabasi, Bursts: The Hidden Pattern Behind Everything We Do (Dutton, New York, 2010).

[33] G.-Y. Chen and L. Saloff-Coste, ALEA, Lat. Am. J. Probab. Math. Stat. 10, 293 (2013).

[34] I. Scholtes, N. Wider, R. Pfitzner, A. Garas, C. J. Tessone, and F. Schweitzer, Nat Commun. 5, 5024 (2014).

[35] A. Jurlewicz and K. Weron, Acta Phys. Pol. B 31, 1077 (2000).

[36] K. S. Cole and R. H. Cole, J. Chem. Phys. 9, 341 (1941).

[37] K. S. Cole and R. H. Cole, J. Chem. Phys. 10, 98 (1942). 